\title{
TAUBERIAN THEOREMS FOR THE WEIGHTED MEAN METHOD OF SUMMABILITY OF INTEGRALS
}

\author{
Fırat Özsaraç and İbrahim Çanak
}

(C) by University of Niš, Serbia | Creative Commons Licence: CC BY-NC-ND Abstract. Let $q$ be a positive weight function on $\mathbf{R}_{+}:=[0, \infty)$ which is integrable in Lebesgue's sense over every finite interval $(0, x)$ for $0<x<\infty$, in symbol: $q \in L_{l o c}^{1}\left(\mathbf{R}_{+}\right)$ such that $Q(x):=\int_{0}^{x} q(t) d t \neq 0$ for each $x>0, Q(0)=0$ and $Q(x) \rightarrow \infty$ as $x \rightarrow \infty$. Given a real or complex-valued function $f \in L_{l o c}^{1}\left(\mathbf{R}_{+}\right)$, we define $s(x):=\int_{0}^{x} f(t) d t$ and

$$
\tau_{q}^{(0)}(x):=s(x), \tau_{q}^{(m)}(x):=\frac{1}{Q(x)} \int_{0}^{x} \tau_{q}^{(m-1)}(t) q(t) d t \quad(x>0, m=1,2, \ldots),
$$

provided that $Q(x)>0$

We say that $\int_{0}^{\infty} f(x) d x$ is summable to $L$ by the $m$-th iteration of weighted mean method determined by the function $q(x)$, or for short, $(\bar{N}, q, m)$ integrable to a finite number $L$ if

$$
\lim _{x \rightarrow \infty} \tau_{q}^{(m)}(x)=L
$$

In this case, we write $s(x) \rightarrow L(\bar{N}, q, m)$.

It is known that if the $\operatorname{limit}_{\lim } \lim _{x \rightarrow \infty} s(x)=L$ exists, then $\lim _{x \rightarrow \infty} \tau_{q}^{(m)}(x)=L$ also exists. However, the converse of this implication is not always true. Some suitable conditions together with the existence of the $\operatorname{limit}_{\lim } \rightarrow \infty \tau_{q}^{(m)}(x)$, which is so called Tauberian conditions, may imply convergence of $\lim _{x \rightarrow \infty} s(x)$.

In this paper, one- and two-sided Tauberian conditions in terms of the generating function and its generalizations for $(\bar{N}, q, m)$ summable integrals of real- or complexvalued functions have been obtained. Some classical type Tauberian theorems given for Cesàro summability $(C, 1)$ and weighted mean method of summability $(\bar{N}, q)$ have been extended and generalized.

Keywords: Tauberian conditions; weight function; summable integrals; finite interval.

\section{Introduction}

Let $q$ be a positive weight function on $\mathbf{R}_{+}:=[0, \infty)$ which is integrable in Lebesgue's sense over every finite interval $(0, x)$ for $0<x<\infty$, in symbol: $q \in$

Received September 17, 2019; accepted November 20, 2019.

2010 Mathematics Subject Classification. Primary 40E05; Secondary 40A10 , 40G05 
$L_{l o c}^{1}\left(\mathbf{R}_{+}\right)$such that $Q(x):=\int_{0}^{x} q(t) d t \neq 0$ for each $x>0, Q(0)=0$ and $Q(x) \rightarrow \infty$ as $x \rightarrow \infty$. Given a real or complex-valued function $f \in L_{l o c}^{1}\left(\mathbf{R}_{+}\right)$, we define $s(x):=\int_{0}^{x} f(t) d t$ and

$$
\tau_{q}^{(0)}(x):=s(x), \tau_{q}^{(m)}(x):=\frac{1}{Q(x)} \int_{0}^{x} \tau_{q}^{(m-1)}(t) q(t) d t \quad(x>0, m=1,2, \ldots),
$$

provided that $Q(x)>0$.

For each integer $m \geq 0$, we define $v_{q}^{(m)}(x)$ by

$$
v_{q}^{(m)}(x)= \begin{cases}\frac{Q(x)}{q(x)} f(x) & , m=0 \\ \frac{1}{Q(x)} \int_{0}^{x} f(t) Q(t) d t & , m=1 \\ \frac{1}{Q(x)} \int_{0}^{x} v_{q}^{(m-1)}(t) q(t) d t & , m \geq 2 .\end{cases}
$$

The identity

$$
\tau_{q}^{(m-1)}(x)-\tau_{q}^{(m)}(x)=v_{q}^{(m)}(x)
$$

is known as the weighted Kronecker identity for the weighted mean method of summability.

It is clear from (1.1) that

$$
\frac{Q(x)}{q(x)} \frac{d}{d x} \tau_{q}^{(m)}(x)=v_{q}^{(m)}(x)
$$

for each integer $m \geq 0$ (see [14]). Here, we call $v_{q}^{(m)}(x)$ the generator of $\tau_{q}^{(m-1)}(x)$ for each integer $m \geq 1$.

We say that $\int_{0}^{\infty} f(x) d x$ is summable to $L$ by the $m$-th iteration of weighted mean method determined by the function $q(x)$, or for short, $(\bar{N}, q, m)$ summable to a finite number $L$ if

$$
\lim _{x \rightarrow \infty} \tau_{q}^{(m)}(x)=L .
$$

It is obvious that $(\bar{N}, q, m)$ summability reduces to the ordinary convergence for $m=0$ and $(\bar{N}, q, 1)$ is the $(\bar{N}, q)$ method. If $q(x)=1$ on $\mathbf{R}_{+}$, then $(\bar{N}, q, m)$ method is the Hölder method of order $m$ and $(\bar{N}, q, 1)$ method is the Cesàro summability method $(C, 1)$.

It is well known that condition $Q(x) \rightarrow \infty$ as $x \rightarrow \infty$ is a necessary and sufficient condition that the existence of the integral

$$
\int_{0}^{\infty} s(x) d x=L
$$

implies (1.2). That is, the $(\bar{N}, q, m)$ summability method is regular, where $m$ is a nonnegative integer. However, the converse of this implication is not always true. Notice that some suitable condition on $s(x)$ together with (1.2) may imply (1.3). 
Such a condition is called a Tauberian condition and resulting theorem is said to be a Tauberian theorem.

Móricz [8] and Fekete and Móricz [6] obtained one-sided and two-sided Tauberian conditions for the weighted mean method $(\bar{N}, q)$ of integrals. Following these works, Totur and Okur [13] proved one-sided boundedness of $v_{q}^{(0)}(x)$ is a Tauberian condition for the weighted mean method of summability $(\bar{N}, q)$ of integrals. From the fact that condition $v_{q}^{(0)}(x) \geq-C$ implies slow decreasing of $s(x)$, Totur and Okur [13] generalized their first result and proved that slow decrease of $s(x)$ is also a Tauberian condition for $(\bar{N}, q)$ method. For a detailed study and some interesting results related to Tauberian theorems for the weighted mean method of summability, we refer the reader to Borwein and Kratz [1], Çanak and Totur [2], Çanak and Totur [3], Çanak and Totur [4], Özsaraç and Çanak [9], Sezer and Çanak [10], Tietz and Zeller [11] and Totur and Çanak [12], etc.

In this paper, one- and two-sided Tauberian conditions in terms of the generating function and its generalizations for summable integrals by $m$-th iteration of weighted means of real- or complex-valued functions have been obtained, respectively. Some classical type Tauberian theorems given for Cesàro summability $(C, 1)$ and weighted mean method of summability $(\bar{N}, q)$ have been extended and generalized.

\section{Main results}

For the main results of the paper, we need the following definitions and notations.

Definition 2.1. ([7]) A positive function $Q$ is called regularly varying of index $\alpha>0$ if

$$
\lim _{x \rightarrow \infty} \frac{Q(\rho x)}{Q(x)}=\rho^{\alpha}, \quad \rho>0 .
$$

It easily follows from Definition 2.1 that for all $\rho>1$ and sufficiently large $x$,

$$
\frac{\rho^{\alpha}}{2\left(\rho^{\alpha}-1\right)} \leq \frac{Q(\rho x)}{Q(\rho x)-Q(x)} \leq \frac{3 \rho^{\alpha}}{2\left(\rho^{\alpha}-1\right)}
$$

and for all $0<\rho<1$ and sufficiently large $x$,

$$
\frac{\rho^{\alpha}}{2\left(1-\rho^{\alpha}\right)} \leq \frac{Q(\rho x)}{Q(x)-Q(\rho x)} \leq \frac{3 \rho^{\alpha}}{2\left(1-\rho^{\alpha}\right)} .
$$

We note that if (2.1) holds, then the following equivalent conditions are clearly satisfied (see [5]):

$$
\liminf _{x \rightarrow \infty} \frac{Q(x)}{Q(\rho x)}<1, \text { for every } \rho>1
$$

and

$$
\liminf _{x \rightarrow \infty} \frac{Q(\rho x)}{Q(x)}<1, \text { for every } 0<\rho<1 .
$$


First, we consider real-valued functions and prove the following Tauberian theorems.

Theorem 2.1. Let (2.1) be satisfied. If a real-valued function $f \in L_{l o c}^{1}\left(\mathbf{R}_{+}\right)$is such that its integral function $s(x)$ is $(\bar{N}, q, m)$ summable to $L$ and $v_{q}^{(m-1)}(x)$ is one-sided bounded, then $s(x)$ is $(\bar{N}, q, m-1)$ summable to $L$.

Corollary 2.1. ([13]) Let (2.1) be satisfied. If a real-valued function $f \in L_{l o c}^{1}\left(\mathbf{R}_{+}\right)$ is such that its integral function $s(x)$ is $(\bar{N}, q, 1)$ summable to $L$ and $v_{q}^{(0)}(x)$ is onesided bounded, then $s(x)$ converges to $L$.

Theorem 2.2. Let (2.4) be satisfied. If a real-valued function $f \in L_{l o c}^{1}\left(\mathbf{R}_{+}\right)$is such that its integral function $s(x)$ is $(\bar{N}, q, m)$ summable to $L$ and $\tau_{q}^{(m-1)}(x)$ is slowly decreasing, then $s(x)$ is $(\bar{N}, q, m-1)$ summable to $L$.

Corollary 2.2. ([13]) Let (2.4) be satisfied. If a real-valued function $f \in L_{l o c}^{1}\left(\mathbf{R}_{+}\right)$ is such that its integral function $s(x)$ is $(\bar{N}, q, 1)$ summable to $L$ and slowly decreasing, then $s(x)$ converges to $L$.

A real-valued function $s(x)$ defined on $\mathbf{R}_{+}$is said to be slowly decreasing if

$$
\lim _{\rho \rightarrow 1^{+}} \liminf _{x \rightarrow \infty} \min _{x \leq t \leq \rho x}(s(t)-s(x)) \geq 0 .
$$

Note that condition (2.6) can be equivalently reformulated as follows:

$$
\lim _{\rho \rightarrow 1^{-}} \liminf _{x \rightarrow \infty} \min _{\rho x \leq t \leq x}(s(x)-s(t)) \geq 0 .
$$

Second, we consider complex-valued functions and prove the following Tauberian theorems.

Theorem 2.3. Let (2.1) be satisfied. If a complex-valued function $f \in L_{l o c}^{1}\left(\mathbf{R}_{+}\right)$ is such that its integral function $s(x)$ is $(\bar{N}, q, m)$ summable to $L$ and $v_{q}^{(m-1)}(x)$ is bounded, then $s(x)$ is $(\bar{N}, q, m-1)$ summable to $L$.

Corollary 2.3. Let (2.1) be satisfied. If a complex-valued function $f \in L_{l o c}^{1}\left(\mathbf{R}_{+}\right)$ is such that its integral function $s(x)$ is $(\bar{N}, q, 1)$ summable to $L$ and $v_{q}^{(0)}(x)$ is bounded, then $s(x)$ converges to $L$.

Theorem 2.4. Let (2.4) be satisfied. If a complex-valued function $f \in L_{l o c}^{1}\left(\mathbf{R}_{+}\right)$ is such that its integral function $s(x)$ is $(\bar{N}, q, m)$ summable to $L$ and $\tau_{q}^{(m-1)}(x)$ is slowly oscillating, then $s(x)$ is $(\bar{N}, q, m-1)$ summable to $L$.

Corollary 2.4. Let (2.4) be satisfied. If a complex-valued function $f \in L_{l o c}^{1}\left(\mathbf{R}_{+}\right)$ is such that its integral function $s(x)$ is $(\bar{N}, q, 1)$ summable to $L$ and slowly oscillating, then $s(x)$ converges to $L$. 
A complex-valued function $s(x)$ defined on $\mathbf{R}_{+}$is said to be slowly oscillating if

$$
\lim _{\rho \rightarrow 1^{+}} \limsup _{x \rightarrow \infty} \max _{x \leq t \leq \rho x}|s(t)-s(x)|=0 .
$$

Note that condition (2.8) can be equivalently reformulated as follows:

$$
\lim _{\rho \rightarrow 1^{-}} \limsup _{x \rightarrow \infty} \max _{\rho x \leq t \leq x}|s(x)-s(t)|=0 .
$$

\section{An auxiliary result}

The following two representations of $s(x)-\tau_{q}^{(1)}(x)$ will be needed in the proofs of our main results.

Lemma 3.1. ([13])

(i) For $\rho>1$ and sufficiently large $x$,

$$
\begin{aligned}
s(x)-\tau_{q}^{(1)}(x) & =\frac{Q(\rho x)}{Q(\rho x)-Q(x)}\left(\tau_{q}^{(1)}(\rho x)-\tau_{q}^{(1)}(x)\right) \\
& -\frac{1}{Q(\rho x)-Q(x)} \int_{x}^{\rho x}(s(t)-s(x)) q(t) d t .
\end{aligned}
$$

(ii) For $0<\rho<1$ and sufficiently large $x$,

$$
\begin{aligned}
s(x)-\tau_{q}^{(1)}(x) & =\frac{Q(\rho x)}{Q(x)-Q(\rho x)}\left(\tau_{q}^{(1)}(x)-\tau_{q}^{(1)}(\rho x)\right) \\
& +\frac{1}{Q(x)-Q(\rho x)} \int_{\rho x}^{x}(s(x)-s(t)) q(t) d t .
\end{aligned}
$$

\section{Proofs of main results}

Proof of Theorem 2.1 Suppose that $s(x)$ is $(\bar{N}, q, m)$ summable to $L$ and $v_{q}^{(m-1)}(x)$ is one-sided bounded. By Lemma 3.1 (i), we have

$$
\begin{aligned}
\tau_{q}^{(m-1)}(x)-\tau_{q}^{(m)}(x) & =\frac{Q(\rho x)}{Q(\rho x)-Q(x)}\left(\tau_{q}^{(m)}(\rho x)-\tau_{q}^{(m)}(x)\right) \\
& -\frac{1}{Q(\rho x)-Q(x)} \int_{x}^{\rho x}\left(\tau_{q}^{(m-1)}(t)-\tau_{q}^{(m-1)}(x)\right) q(t) d t \\
& =\frac{Q(\rho x)}{Q(\rho x)-Q(x)}\left(\tau_{q}^{(m)}(\rho x)-\tau_{q}^{(m)}(x)\right) \\
& -\frac{1}{Q(\rho x)-Q(x)} \int_{x}^{\rho x}\left(\int_{x}^{t} \frac{d}{d z} \tau_{q}^{(m-1)}(z) d z\right) q(t) d t .
\end{aligned}
$$


Since $v_{q}^{(m-1)}(x)$ is one-sided bounded, we get

$$
\begin{aligned}
\tau_{q}^{(m-1)}(x)-\tau_{q}^{(m)}(x) & \leq \frac{Q(\rho x)}{Q(\rho x)-Q(x)}\left(\tau_{q}^{(m)}(\rho x)-\tau_{q}^{(m)}(x)\right) \\
& +\frac{C}{Q(\rho x)-Q(x)} \int_{x}^{\rho x}\left(\int_{x}^{t} \frac{q(z)}{Q(z)} d z\right) q(t) d t \\
& =\frac{Q(\rho x)}{Q(\rho x)-Q(x)}\left(\tau_{q}^{(m)}(\rho x)-\tau_{q}^{(m)}(x)\right) \\
& +\frac{C}{Q(\rho x)-Q(x)} \int_{x}^{\rho x} q(t) \log \frac{Q(t)}{Q(x)} d t \\
& =\frac{Q(\rho x)}{Q(\rho x)-Q(x)}\left(\tau_{q}^{(m)}(\rho x)-\tau_{q}^{(m)}(x)\right)+C \log \frac{Q(\rho x)}{Q(x)} .
\end{aligned}
$$

By $(2.2)$ and $(\bar{N}, q, m)$ summability of $s(x)$, we have

$$
\lim _{x \rightarrow \infty} \frac{Q(\rho x)}{Q(\rho x)-Q(x)}\left(\tau_{q}^{(m)}(\rho x)-\tau_{q}^{(m)}(x)\right)=0 .
$$

Taking (4.2) into account in (4.1), we obtain

$$
\limsup _{x \rightarrow \infty}\left(\tau_{q}^{(m-1)}(x)-\tau_{q}^{(m)}(x)\right) \leq \limsup _{x \rightarrow \infty}\left(C \log \frac{Q(\rho x)}{Q(x)}\right)=C \log \rho^{\alpha} .
$$

Letting $\rho \rightarrow 1^{+}$in the last inequality, we have

$$
\limsup _{x \rightarrow \infty}\left(\tau_{q}^{(m-1)}(x)-\tau_{q}^{(m)}(x)\right) \leq 0 .
$$

Similarly, from Lemma 3.1 (ii), we have

$$
\begin{aligned}
\tau_{q}^{(m-1)}(x)-\tau_{q}^{(m)}(x) & =\frac{Q(\rho x)}{Q(x)-Q(\rho x)}\left(\tau_{q}^{(m)}(x)-\tau_{q}^{(m)}(\rho x)\right) \\
& +\frac{1}{Q(x)-Q(\rho x)} \int_{\rho x}^{x}\left(\tau_{q}^{(m-1)}(x)-\tau_{q}^{(m-1)}(t)\right) q(t) d t \\
& =\frac{Q(\rho x)}{Q(x)-Q(\rho x)}\left(\tau_{q}^{(m)}(x)-\tau_{q}^{(m)}(\rho x)\right) \\
& +\frac{1}{Q(x)-Q(\rho x)} \int_{\rho x}^{x}\left(\int_{t}^{x} \frac{d}{d z} \tau_{q}^{(m-1)}(z) d z\right) q(t) d t .
\end{aligned}
$$

Since $v_{q}^{(m-1)}(x)$ is one-sided bounded, we get

$$
\tau_{q}^{(m-1)}(x)-\tau_{q}^{(m)}(x) \geq \frac{Q(\rho x)}{Q(x)-Q(\rho x)}\left(\tau_{q}^{(m)}(x)-\tau_{q}^{(m)}(\rho x)\right)
$$




$$
\begin{aligned}
& -\frac{C}{Q(x)-Q(\rho x)} \int_{\rho x}^{x}\left(\int_{t}^{x} \frac{q(z)}{Q(z)} d z\right) q(t) d t \\
& =\frac{Q(\rho x)}{Q(x)-Q(\rho x)}\left(\tau_{q}^{(m)}(x)-\tau_{q}^{(m)}(\rho x)\right) \\
& -\frac{C}{Q(x)-Q(\rho x)} \int_{\rho x}^{x} q(t) \log \frac{Q(x)}{Q(t)} d t \\
& =\frac{Q(\rho x)}{Q(x)-Q(\rho x)}\left(\tau_{q}^{(m)}(x)-\tau_{q}^{(m)}(\rho x)\right)-C \log \frac{Q(x)}{Q(\rho x)} .
\end{aligned}
$$

By $(2.3)$ and $(\bar{N}, q, m)$ summability of $s(x)$, we obtain

$$
\lim _{x \rightarrow \infty} \frac{Q(\rho x)}{Q(x)-Q(\rho x)}\left(\tau_{q}^{(m)}(x)-\tau_{q}^{(m)}(\rho x)\right)=0 .
$$

Taking (4.5) into account in (4.4), we obtain

$$
\limsup _{x \rightarrow \infty}\left(\tau_{q}^{(m-1)}(x)-\tau_{q}^{(m)}(x)\right) \geq-\liminf _{x \rightarrow \infty}\left(C \log \frac{Q(x)}{Q(\rho x)}\right)=-C \log \rho^{\alpha} .
$$

Letting $\rho \rightarrow 1^{-}$in the last inequality, we have

$$
\limsup _{x \rightarrow \infty}\left(\tau_{q}^{(m-1)}(x)-\tau_{q}^{(m)}(x)\right) \geq 0
$$

Combining (4.3) and (4.6), we obtain $s(x)$ is $(\bar{N}, q, m-1)$ summable to $L$.

Proof of Theorem 2.2 Suppose that $s(x)$ is $(\bar{N}, q, m)$ summable to $L$ and $\tau_{q}^{(m-1)}(x)$ is slowly decreasing. By Lemma 3.1 (i), we have

$$
\begin{aligned}
\tau_{q}^{(m-1)}(x)-\tau_{q}^{(m)}(x) & =\frac{Q(\rho x)}{Q(\rho x)-Q(x)}\left(\tau_{q}^{(m)}(\rho x)-\tau_{q}^{(m)}(x)\right) \\
& -\frac{1}{Q(\rho x)-Q(x)} \int_{x}^{\rho x}\left(\tau_{q}^{(m-1)}(t)-\tau_{q}^{(m-1)}(x)\right) q(t) d t \\
& \leq \frac{Q(\rho x)}{Q(\rho x)-Q(x)}\left(\tau_{q}^{(m)}(\rho x)-\tau_{q}^{(m)}(x)\right) \\
& -\frac{1}{Q(\rho x)-Q(x)} \int_{x}^{\rho x} q(t) \min _{x \leq t \leq \rho x}\left(\tau_{q}^{(m-1)}(t)-\tau_{q}^{(m-1)}(x)\right) d t \\
& =\frac{Q(\rho x)}{Q(\rho x)-Q(x)}\left(\tau_{q}^{(m)}(\rho x)-\tau_{q}^{(m)}(x)\right) \\
& -\min _{x \leq t \leq \rho x}\left(\tau_{q}^{(m-1)}(t)-\tau_{q}^{(m-1)}(x)\right) .
\end{aligned}
$$


Taking the lim sup of both sides of (4.7), we get

$$
\begin{array}{ll}
\limsup _{x \rightarrow \infty}\left(\tau_{q}^{(m-1)}(x)-\tau_{q}^{(m)}(x)\right) & \leq \limsup _{x \rightarrow \infty} \frac{Q(\rho x)}{Q(\rho x)-Q(x)}\left(\tau_{q}^{(m)}(\rho x)-\tau_{q}^{(m)}(x)\right) \\
& -\liminf _{x \rightarrow \infty} \min _{x \leq t \leq \rho x}\left(\tau_{q}^{(m-1)}(t)-\tau_{q}^{(m-1)}(x)\right) .
\end{array}
$$

By (2.4), we have

$$
0<\limsup _{x \rightarrow \infty} \frac{Q(\rho x)}{Q(\rho x)-Q(x)}=1+\left(\liminf _{x \rightarrow \infty} \frac{Q(\rho x)}{Q(x)}-1\right)^{-1}<\infty .
$$

Since $s(x)$ is $(\bar{N}, q, m)$ summable to $L$, the first term on the right-hand side vanishes in (4.8). From this, we obtain

$$
\limsup _{x \rightarrow \infty}\left(\tau_{q}^{(m-1)}(x)-\tau_{q}^{(m)}(x)\right) \leq-\liminf _{x \rightarrow \infty} \min _{x \leq t \leq \rho x}\left(\tau_{q}^{(m-1)}(t)-\tau_{q}^{(m-1)}(x)\right) .
$$

Taking the limit of (4.8) as $\rho \rightarrow 1^{+}$, we have

$$
\limsup _{x \rightarrow \infty}\left(\tau_{q}^{(m-1)}(x)-\tau_{q}^{(m)}(x)\right) \leq 0 .
$$

Similarly, by Lemma 3.1 (ii), we have

$$
\begin{aligned}
\tau_{q}^{(m-1)}(x)-\tau_{q}^{(m)}(x) & =\frac{Q(\rho x)}{Q(x)-Q(\rho x)}\left(\tau_{q}^{(m)}(x)-\tau_{q}^{(m)}(\rho x)\right) \\
& +\frac{1}{Q(x)-Q(\rho x)} \int_{\rho x}^{x}\left(\tau_{q}^{(m-1)}(x)-\tau_{q}^{(m-1)}(t)\right) q(t) d t \\
& \geq \frac{Q(\rho x)}{Q(x)-Q(\rho x)}\left(\tau_{q}^{(m)}(x)-\tau_{q}^{(m)}(\rho x)\right) \\
& +\frac{1}{Q(x)-Q(\rho x)} \int_{\rho x}^{x} q(t) \min _{\rho x \leq t \leq x}\left(\tau_{q}^{(m-1)}(x)-\tau_{q}^{(m-1)}(t)\right) d t \\
& =\frac{Q(\rho x)}{Q(x)-Q(\rho x)}\left(\tau_{q}^{(m)}(x)-\tau_{q}^{(m)}(\rho x)\right) \\
& +\min _{\rho x \leq t \leq x}\left(\tau_{q}^{(m-1)}(x)-\tau_{q}^{(m-1)}(t)\right) .
\end{aligned}
$$

From (4.10), we get

$$
\begin{array}{ll}
\liminf _{x \rightarrow \infty}\left(\tau_{q}^{(m-1)}(x)-\tau_{q}^{(m)}(x)\right) & \geq \liminf _{x \rightarrow \infty} \frac{Q(\rho x)}{Q(x)-Q(\rho x)}\left(\tau_{q}^{(m)}(x)-\tau_{q}^{(m)}(\rho x)\right) \\
& +\liminf _{x \rightarrow \infty} \min _{\rho x \leq t \leq x}\left(\tau_{q}^{(m-1)}(x)-\tau_{q}^{(m-1)}(t)\right) .
\end{array}
$$


By (2.4), we have

$$
0<\liminf _{x \rightarrow \infty} \frac{Q(\rho x)}{Q(x)-Q(\rho x)}=\left(\limsup _{x \rightarrow \infty} \frac{Q(x)}{Q(\rho x)}-1\right)^{-1}<\infty .
$$

Since $s(x)$ is $(\bar{N}, q, m)$ summable to $L$, the first term on the right-hand side vanishes in (4.11). From this, we obtain

$$
\liminf _{x \rightarrow \infty}\left(\tau_{q}^{(m-1)}(x)-\tau_{q}^{(m)}(x)\right) \geq \liminf _{x \rightarrow \infty} \min _{\rho x \leq t \leq x}\left(\tau_{q}^{(m-1)}(x)-\tau_{q}^{(m-1)}(t)\right) .
$$

Taking the limit of (4.11) as $\rho \rightarrow 1^{-}$, we have

$$
\liminf _{x \rightarrow \infty}\left(\tau_{q}^{(m-1)}(x)-\tau_{q}^{(m)}(x)\right) \geq 0 .
$$

Combining (4.9) and (4.12), we obtain $s(x)$ is $(\bar{N}, q, m-1)$ summable to $L$.

Proof of Theorem 2.3 Suppose that $s(x)$ is $(\bar{N}, q, m)$ summable to $L$ and $v_{q}^{(m-1)}(x)$ is bounded. By Lemma 3.1 (i), we have

$$
\begin{aligned}
\left|\tau_{q}^{(m-1)}(x)-\tau_{q}^{(m)}(x)\right| & \leq \frac{Q(\rho x)}{Q(\rho x)-Q(x)}\left|\tau_{q}^{(m)}(\rho x)-\tau_{q}^{(m)}(x)\right| \\
& +\frac{1}{Q(\rho x)-Q(x)} \int_{x}^{\rho x}\left|\tau_{q}^{(m-1)}(t)-\tau_{q}^{(m-1)}(x)\right| q(t) d t \\
& =\frac{Q(\rho x)}{Q(\rho x)-Q(x)}\left|\tau_{q}^{(m)}(\rho x)-\tau_{q}^{(m)}(x)\right| \\
& +\frac{1}{Q(\rho x)-Q(x)} \int_{x}^{\rho x}\left|\int_{x}^{t} \frac{d}{d z} \tau_{q}^{(m-1)}(z) d z\right| q(t) d t .
\end{aligned}
$$

Since $v_{q}^{(m-1)}(x)$ is bounded, we get

$$
\begin{aligned}
\left|\tau_{q}^{(m-1)}(x)-\tau_{q}^{(m)}(x)\right| & \leq \frac{Q(\rho x)}{Q(\rho x)-Q(x)}\left|\tau_{q}^{(m)}(\rho x)-\tau_{q}^{(m)}(x)\right| \\
& +\frac{C}{Q(\rho x)-Q(x)} \int_{x}^{\rho x}\left|\int_{x}^{t} \frac{q(z)}{Q(z)} d z\right| q(t) d t \\
& =\frac{Q(\rho x)}{Q(\rho x)-Q(x)}\left|\tau_{q}^{(m)}(\rho x)-\tau_{q}^{(m)}(x)\right| \\
& +\frac{C}{Q(\rho x)-Q(x)} \int_{x}^{\rho x} q(t) \log \frac{Q(t)}{Q(x)} d t \\
& \leq \frac{Q(\rho x)}{Q(\rho x)-Q(x)}\left|\tau_{q}^{(m)}(\rho x)-\tau_{q}^{(m)}(x)\right|+C \log \frac{Q(\rho x)}{Q(x)}
\end{aligned}
$$


By $(2.2)$ and $(\bar{N}, q, m)$ summability of $s(x)$, we have

$$
\lim _{x \rightarrow \infty} \frac{Q(\rho x)}{Q(\rho x)-Q(x)}\left|\tau_{q}^{(m)}(\rho x)-\tau_{q}^{(m)}(x)\right|=0 .
$$

Taking the lim sup of both sides of (4.13) gives

$$
\limsup _{x \rightarrow \infty}\left|\tau_{q}^{(m-1)}(x)-\tau_{q}^{(m)}(x)\right| \leq \limsup _{x \rightarrow \infty}\left(C \log \frac{P(\rho x)}{Q(x)}\right)=C \log \rho^{\alpha} .
$$

Letting $\rho \rightarrow 1^{+}$in last inequality, we have

$$
\limsup _{x \rightarrow \infty}\left|\tau_{q}^{(m-1)}(x)-\tau_{q}^{(m)}(x)\right| \leq 0 .
$$

Similarly, from Lemma 3.1 (ii), we have

$$
\begin{aligned}
\left|\tau_{q}^{(m-1)}(x)-\tau_{q}^{(m)}(x)\right| & \leq \frac{P(\rho x)}{Q(x)-Q(\rho x)}\left|\tau_{q}^{(m)}(x)-\tau_{q}^{(m)}(\rho x)\right| \\
& +\frac{1}{Q(x)-Q(\rho x)} \int_{\rho x}^{x}\left|\tau_{q}^{(m-1)}(x)-\tau_{q}^{(m-1)}(t)\right| q(t) d t \\
& =\frac{Q(\rho x)}{Q(x)-Q(\rho x)}\left|\tau_{q}^{(m)}(x)-\tau_{q}^{(m)}(\rho x)\right| \\
& +\frac{1}{Q(x)-Q(\rho x)} \int_{\rho x}^{x}\left|\int_{t}^{x} \frac{d}{d z} \tau_{q}^{(m-1)}(z) d z\right| q(t) d t .
\end{aligned}
$$

Since $v_{q}^{(m-1)}(x)$ is bounded, we get

$$
\begin{aligned}
\left|\tau_{q}^{(m-1)}(x)-\tau_{q}^{(m)}(x)\right| & \leq \frac{P(\rho x)}{Q(x)-Q(\rho x)}\left|\tau_{q}^{(m)}(x)-\tau_{q}^{(m)}(\rho x)\right| \\
& +\frac{C}{Q(x)-Q(\rho x)} \int_{\rho x}^{x}\left|\int_{t}^{x} \frac{p(z)}{P(z)} d z\right| q(t) d t \\
& =\frac{Q(\rho x)}{Q(x)-Q(\rho x)}\left|\tau_{q}^{(m)}(x)-\tau_{q}^{(m)}(\rho x)\right| \\
& +\frac{C}{Q(x)-Q(\rho x)} \int_{\rho x}^{x} q(t) \log \frac{Q(x)}{Q(t)} d t \\
& \leq \frac{Q(\rho x)}{Q(x)-Q(\rho x)}\left|\tau_{q}^{(m)}(x)-\tau_{q}^{(m)}(\rho x)\right|+C \log \frac{Q(x)}{Q(\rho x)} .
\end{aligned}
$$

By $(2.3)$ and $(\bar{N}, p, m)$ summability of $s(x)$, we have

$$
\lim _{x \rightarrow \infty} \frac{Q(\rho x)}{P(x)-Q(\rho x)}\left|\tau_{q}^{(m)}(x)-\tau_{q}^{(m)}(\rho x)\right|=0 .
$$


From (4.15), we get

$$
\limsup _{x \rightarrow \infty}\left|\tau_{q}^{(m-1)}(x)-\tau_{q}^{(m)}(x)\right| \leq \limsup _{x \rightarrow \infty}\left(C \log \frac{Q(x)}{Q(\rho x)}\right)=C \log \rho^{\alpha} .
$$

Letting $\rho \rightarrow 1^{-}$in last inequality, we have

$$
\limsup _{x \rightarrow \infty}\left|\tau_{q}^{(m-1)}(x)-\tau_{q}^{(m)}(x)\right| \leq 0
$$

From either (4.14) or (4.16), we conclude $s(x)$ is $(\bar{N}, q, m-1)$ summable to $L$.

Proof of Theorem 2.4 Suppose that $s(x)$ is $(\bar{N}, q, m)$ summable to $L$ and $\tau_{q}^{(m-1)}(x)$ is slowly oscillating. By Lemma 3.1 (i), we have

$$
\begin{aligned}
\left|\tau_{q}^{(m-1)}(x)-\tau_{q}^{(m)}(x)\right| & =\frac{Q(\rho x)}{Q(\rho x)-Q(x)}\left|\tau_{q}^{(m)}(\rho x)-\tau_{q}^{(m)}(x)\right| \\
& +\frac{1}{Q(\rho x)-Q(x)} \int_{x}^{\rho x}\left|\tau_{q}^{(m-1)}(t)-\tau_{q}^{(m-1)}(x)\right| q(t) d t \\
& \leq \frac{Q(\rho x)}{Q(\rho x)-Q(x)}\left|\tau_{q}^{(m)}(\rho x)-\tau_{q}^{(m)}(x)\right| \\
& +\frac{1}{Q(\rho x)-Q(x)} \int_{x}^{\rho(t)} \max _{x \leq t \leq \rho x}\left(\left|\tau_{q}^{(m-1)}(t)-\tau_{q}^{(m-1)}(x)\right|\right) d t \\
& \leq \frac{Q(\rho x)}{Q(\rho x)-Q(x)}\left|\tau_{q}^{(m)}(\rho x)-\tau_{q}^{(m)}(x)\right| \\
& +\max _{x \leq t \leq \rho x}\left|\tau_{q}^{(m-1)}(t)-\tau_{q}^{(m-1)}(x)\right| .
\end{aligned}
$$

From (4.17), we get

$$
\begin{array}{ll}
\limsup _{x \rightarrow \infty}\left|\tau_{q}^{(m-1)}(x)-\tau_{q}^{(m)}(x)\right| & \leq \limsup _{x \rightarrow \infty} \frac{Q(\rho x)}{Q(\rho x)-Q(x)}\left|\tau_{q}^{(m)}(\rho x)-\tau_{q}^{(m)}(x)\right| \\
& +\limsup _{x \rightarrow \infty} \max _{x \leq t \leq \rho x}\left|\tau_{q}^{(m-1)}(t)-\tau_{q}^{(m-1)}(x)\right| .
\end{array}
$$

By (2.4), we have

$$
0<\limsup _{x \rightarrow \infty} \frac{Q(\rho x)}{Q(\rho x)-Q(x)}=1+\left(\liminf _{x \rightarrow \infty} \frac{Q(\rho x)}{Q(x)}-1\right)^{-1}<\infty .
$$

Since $s(x)$ is $(\bar{N}, q, m)$ summable to $L$, the first term on the right side vanishes in (4.18). From this, we obtain

$$
\limsup _{x \rightarrow \infty}\left|\tau_{q}^{(m-1)}(x)-\tau_{q}^{(m)}(x)\right| \leq \limsup _{x \rightarrow \infty} \max _{x \leq t \leq \rho x}\left|\tau_{q}^{(m-1)}(t)-\tau_{q}^{(m-1)}(x)\right| .
$$


Taking the limit of (4.18) as $\rho \rightarrow 1^{+}$, we have

$$
\limsup _{x \rightarrow \infty}\left|\tau_{q}^{(m-1)}(x)-\tau_{q}^{(m)}(x)\right| \leq 0 .
$$

Similarly, by Lemma 3.1 (ii), we have

$$
\begin{aligned}
\left|\tau_{q}^{(m-1)}(x)-\tau_{q}^{(m)}(x)\right| & =\frac{Q(\rho x)}{Q(x)-Q(\rho x)}\left|\tau_{q}^{(m)}(x)-\tau_{q}^{(m)}(\rho x)\right| \\
& +\frac{1}{Q(x)-Q(\rho x)} \int_{\rho x}^{x}\left|\tau_{q}^{(m-1)}(x)-\tau_{q}^{(m-1)}(t)\right| q(t) d t \\
& \leq \frac{Q(\rho x)}{Q(x)-Q(\rho x)}\left|\tau_{q}^{(m)}(x)-\tau_{q}^{(m)}(\rho x)\right| \\
& +\frac{1}{Q(x)-Q(\rho x)} \int_{\rho x}^{x} q(t) \max _{\rho x \leq t \leq x}\left(\left|\tau_{q}^{(m-1)}(x)-\tau_{q}^{(m-1)}(t)\right|\right) d t \\
& \leq \frac{Q(\rho x)}{Q(x)-Q(\rho x)}\left|\tau_{q}^{(m)}(x)-\tau_{q}^{(m)}(\rho x)\right| \\
& +\max _{\rho x \leq t \leq x}\left|\tau_{q}^{(m-1)}(x)-\tau_{q}^{(m-1)}(t)\right| .
\end{aligned}
$$

From (4.20), we get

$$
\begin{aligned}
\limsup _{x \rightarrow \infty}\left|\tau_{q}^{(m-1)}(x)-\tau_{q}^{(m)}(x)\right| & \leq \limsup _{x \rightarrow \infty} \frac{Q(\rho x)}{Q(x)-Q(\rho x)}\left|\tau_{q}^{(m)}(x)-\tau_{q}^{(m)}(\rho x)\right| \\
& +\limsup _{x \rightarrow \infty} \max _{\rho x \leq t \leq x}\left|\tau_{q}^{(m-1)}(x)-\tau_{q}^{(m-1)}(t)\right| .
\end{aligned}
$$

By (2.4), we have

$$
0<\liminf _{x \rightarrow \infty} \frac{Q(\rho x)}{Q(x)-Q(\rho x)}=\left(\limsup _{x \rightarrow \infty} \frac{Q(x)}{Q(\rho x)}-1\right)^{-1}<\infty .
$$

Since $s(x)$ is $(\bar{N}, q, m)$ summable to $L$, the first term on the right-hand side vanishes in (4.21). From this, we obtain

$$
\limsup _{x \rightarrow \infty}\left|\tau_{q}^{(m-1)}(x)-\tau_{q}^{(m)}(x)\right| \leq \limsup _{x \rightarrow \infty} \max _{\rho x \leq t \leq x}\left|\tau_{q}^{(m-1)}(x)-\tau_{q}^{(m-1)}(t)\right| .
$$

Taking the limit of (4.21) as $\rho \rightarrow 1^{-}$, we have

$$
\limsup _{x \rightarrow \infty}\left|\tau_{q}^{(m-1)}(x)-\tau_{q}^{(m)}(x)\right| \leq 0 .
$$

From either (4.19) or (4.22), we conclude $s(x)$ is $(\bar{N}, q, m-1)$ summable to $L$. 


\section{Conclusion}

In this paper, we introduce Tauberian conditions in terms of the generator and its generalizations for summable integrals by $m$-th iteration of weighted means of real- or complex-valued functions, respectively. Tauberian conditions for summable double integrals by $m$-th iteration of weighted means of real- or complex-valued functions will be illustrated in a forthcoming work.

\section{REF E R E N C E S}

1. D. Borwein and W. KRATz, W: On relations between weighted mean and power series methods of summability. J. Math. Anal. Appl. 139 (1989), 178-186.

2. I. ÇANAK and Ü. Totur: Some Tauberian theorems for the weighted mean methods of summability. Comput. Math. Appl. 62 (2011), 2609-2615.

3. I. ÇANAK and Ü. ToTUR: Extended Tauberian theorem for the weighted mean method of summability. Ukrainian Math. J. 65 (2013), 1032-1041.

4. I. ÇANAK and Ü. Totur: A theorem for the $(J, p)$ summability method. Acta Math. Hungar. 145 (2015), 220-228.

5. C. Chen and J. Hsu: Tauberian theorems for weighted means of double sequences. Anal. Math. 26 (2000), 243-262.

6. Á. FEKETE and F. MórICZ: Necessary and sufficient Tauberian conditions in the case of weighted mean summable integrals over $R_{+}$. II. Publ. Math. Debrecen. 67 (2005), $65-78$.

7. J. KARAmata: Sur un mode de croissance régulière. Théorèmes fondamentaux. Bull. Soc. Math. France. 61 (1933), 55-62.

8. F. MóRICZ: Ordinary convergence follows from statistical summability $(C, 1)$ in the case of slowly decreasing or oscillating sequences. Colloq. Math. 99 (2004), 207-219.

9. F. ÖZSARAÇ and İ. ÇANAK: Tauberian theorems for iterations of weighted mean summable integrals. Positivity. 23 (2019), 219-231.

10. S. A. SEzER and I. ÇANAK: On a Tauberian theorem for the weighted mean method of summability. Kuwait J. Sci. 42 (2015), 1-9.

11. H. Tietz and K. Zeller: Tauber-Sätze für bewichtete Mittel. Arch. Math. (Basel). 68 (1997), 214-220.

12. Ü. TOTUR and I. ÇANAK: Some general Tauberian conditions for the weighted mean summability method. Comput. Math. Appl. 63 (2012), 999-1006.

13. Ü. TOTUR and M. A. OKuR: Alternative proofs of some classical Tauberian theorems for the weighted mean method of integrals. Filomat. 29 (2015), 2281-2287.

14. Ü. Totur, M. A. Okur and İ. ÇAnAK: One-sided Tauberian conditions for the $(\bar{N}, p)$ summability of integrals. Politehn. Univ. Bucharest Sci. Bull. Ser. A Appl. Math. Phys. 80 (2018), 65-74. 
Firat Özsaraç

Kırıkkale University

Department of Mathematics

Kırıkkale, Turkey

firatozsarac@kku.edu.tr

İbrahim Çanak

Ege University

Department of Mathematics

İzmir, Turkey

ibrahim.canak@ege.edu.tr 